\title{
Van der Waals-Like Phase Transition from Holographic Entanglement Entropy in Lorentz Breaking Massive Gravity
}

\author{
Xian-Ming Liu, ${ }^{1}$ Hong-Bo Shao, ${ }^{2}$ and Xiao-Xiong Zeng ${ }^{3}$ \\ ${ }^{1}$ School of Science, Hubei University for Nationalities, Enshi 445000, China \\ ${ }^{2}$ College of Science, Agricultural University of Hebei, Baoding 071000, China \\ ${ }^{3}$ School of Material Science and Engineering, Chongqing Jiaotong University, Chongqing 400074, China \\ Correspondence should be addressed to Xiao-Xiong Zeng; xxzengphysics@163.com
}

Received 12 June 2017; Revised 15 August 2017; Accepted 24 August 2017; Published 2 October 2017

Academic Editor: Li Li

Copyright (C) 2017 Xian-Ming Liu et al. This is an open access article distributed under the Creative Commons Attribution License, which permits unrestricted use, distribution, and reproduction in any medium, provided the original work is properly cited. The publication of this article was funded by SCOAP ${ }^{3}$.

\begin{abstract}
Phase transition of AdS black holes in Lorentz breaking massive gravity has been studied in the framework of holography. We find that there is a first-order phase transition (FPT) and second-order phase transition (SPT) both in Bekenstein-Hawking entropy(BHE-) temperature plane and in holographic entanglement entropy- (HEE-) temperature plane. Furthermore, for the FPT, the equal area law is checked and for the SPT, the critical exponent of the heat capacity is also computed. Our results confirm that the phase structure of HEE is similar to that of BHE in Lorentz breaking massive gravity, which implies that HEE and BHE have some potential underlying relationship.
\end{abstract}

\section{Introduction}

The study of HEE and quantum phase transitions of black holes has attracted a lot of interest in recent years. On one hand, HEE can be used as a perfect probe to study quantum information science [1-3], strongly correlated quantum systems [4-13], and Many-Body Systems [14, 15]. On the other hand, investigation on HEE of black holes may shed some light on understanding the nature of BHE $[16,17]$.

Nearly ten years ago, a holographic derivation of the HEE in conformal quantum field theories was proposed by Ryu and Takayanagi using the famous AdS/CFT correspondence $[18,19]$. Recently the HEE has been used as a probe to investigate the phase structure of the Reissner-Nordstrom AdS black hole [20]. The results showed that there is a Van der Waals-like (VDW) phase transition at the same critical temperature in both the fixed charge ensemble and chemical potential ensemble in the HEE-temperature plane. They also found that the SPT occurs for the HEE at the same critical point as the BHE with nearly the same critical exponent. This work was soon generalized to the extended phase space where the cosmological constant is considered as a thermodynamical variable [21]. Very recently, the equal area law of HEE was proved to hold for the FPT in the HEEtemperature plane [22]. Based on [20], VDW phase transition of HEE in various AdS black holes has been studied in [2332]. All of these works showed that the HEE undergoes the same VDW phase transition as that of the BHE.

Massive gravity theories have attracted considerable interest recently. One of these reasons is that these alternative theories of gravity could explain the accelerated expansion of the universe without dark energy. The graviton behaves like a lattice excitation and exhibits a Drude peak in this theory. Current experimental data from the observation of gravitational waves by advanced LIGO require the graviton mass to be smaller than the inverse period of orbital motion of the binary system; that is, $m_{g}<1.2 \times 10^{-22} \mathrm{eV} / c^{2}$ [33]. Another important reason for the interest in massive gravity is that the possibility of the mass graviton could help to understand the quantum gravity effect. The first to introduce a mass to the graviton is in [34]. However this primitive linear massive gravity theory contains the socalled Boulware-Deser ghosts problem [35] that was solved by a nonlinear massive gravity theory $[36,37]$, where the 
mass terms are obtained by introducing a reference metric. Recently Vegh proposed a new reference metric to describe a class of strongly interacting quantum field theories with broken translational symmetry in the holographic framework [38]. The recent progress in massive gravity can be found in $[39,40]$.

Here, we consider AdS black holes in Lorentz breaking massive gravity. In the massive gravity, the graviton acquires a mass by Lorentz symmetry breaking, which is very similar to the Higgs mechanism. A review of Lorentz-violating massive gravity theory can be found in $[41,42]$. In this paper, we focus on the study of the VDW phase transition of AdS black holes in Lorentz breaking massive gravity using the HEE. The main motivation of this paper is to explore whether the BHE phase transition can also be described by HEE in Lorentz breaking massive gravity. Firstly, we would like to extend proposals in [20] to study VDW phase transitions in AdS black hole with a spherical horizon in Lorentz-violating massive gravity with the HEE as a probe. What is more, we also would like to check Maxwell's equal area law and critical exponent of the heat capacity, which are two characteristic quantities in VDW phase transition.

The organization of this paper is as follows. In the next section, we shall provide a brief review of the black hole solution in Lorentz breaking massive gravity firstly. Then we will study the VDW phase transitions and critical phenomena for the AdS black hole in the BHE-temperature plane. In Section 3, we mainly concentrate on the VDW phase transition and critical phenomena in the framework of HEE. The last section is devoted to our discussions and conclusions.

\section{Phase Transition and Critical Phenomena of AdS Black Holes in Lorentz Breaking Massive Gravity}

2.1. Review of AdS Black Holes in Lorentz Breaking Massive Gravity. The four-dimensional Lorentz breaking massive gravity can be obtained by adding nonderivative coupling scalar fields to the standard Einstein gravity theory. As a matter field is considered, the theory can be described by the following action $[41,42]$ :

$$
S=\int d^{4} x \sqrt{-g}\left[-M_{\mathrm{Pl}}^{2} R+L_{m}+\ell^{4} \Psi\left(X, \Pi^{i j}\right)\right]
$$

here the first two terms are the curvature and ordinary matter minimally coupled to gravity, respectively, and the third term $\Psi$ contains two functions $X$ and $\Pi^{i j}$ which relate to the four scalar fields, $\Xi^{0}$ and $\Xi^{i}$ as

$$
\begin{aligned}
X & =\frac{\partial^{\mu} \Xi^{0} \partial_{\mu} \Xi^{0}}{\ell^{4}}, \\
\Pi^{i j} & =\frac{\partial^{\mu} \Xi^{i} \partial_{\mu} \Xi^{j}}{\ell^{4}}-\frac{\partial^{\mu} \Xi^{i} \partial_{\mu} \Xi^{0} \partial^{\nu} \Xi^{j} \partial_{\nu} \Xi^{0}}{\ell^{8} X} .
\end{aligned}
$$

When the four scalar fields get a space-time depending vacuum expectation value, the system will break the Lorentz symmetry. What is more, the action can also be taken as a low-energy effective theory with the ultraviolet cutoff scale $\ell$. Here the scale parameter $\ell$ has the dimension of mass and is in the order of $\sqrt{m_{g} M_{\mathrm{Pl}}}$, where $m_{g}$ and $M_{\mathrm{Pl}}$ are the graviton mass and the Plank mass, respectively.

The AdS black hole solutions can be obtained from the above theory $[43,44]$. The metric corresponding to the AdS black holes is given by

$$
d s^{2}=-f(r) d t^{2}+f(r)^{-1} d r^{2}+r^{2}\left(d \varphi^{2}+\sin ^{2} \varphi d \phi^{2}\right),
$$

with

$$
f(r)=1-\frac{2 M}{r}-\gamma \frac{Q^{2}}{r^{\lambda}}-\frac{\Lambda r^{2}}{3} .
$$

Here, the four scalar fields, $\Xi^{0}$ and $\Xi^{i}$, for this particular solution are given by

$$
\begin{aligned}
& \Xi^{0}=\ell^{2}(t+\eta(r)), \\
& \Xi^{i}=\ell^{2} \alpha x^{i},
\end{aligned}
$$

in which

$$
\begin{aligned}
\eta(r) & = \pm \int \frac{d r}{f(r)} \\
& {\left[1-f(r)\left(\frac{\gamma Q^{2} \lambda(\lambda-1)}{12 m_{g}^{2} \alpha^{6}} \frac{1}{r^{\lambda+1}}+1\right)^{-1}\right]^{1 / 2}, }
\end{aligned}
$$

in which the scalar charge $Q$ is related to massive gravity and the constant $\alpha$ which is determined by the cosmological constant $\Lambda$ and the graviton mass $m_{g}$ with the relation $\Lambda=$ $2 m_{g}^{2}\left(1-\alpha^{6}\right)$. In this paper, we will set $\alpha>1$ such that $\Lambda<0$ leading to Anti-de Sitter black holes. The constant $\lambda$ is a positive integration constant. When $\lambda<1$, the ADM mass of the black hole solution diverges. For $\lambda>1$, the metric approaches the Schwarzschild-AdS black holes with a finite mass $M$ as $r \rightarrow \infty$. Thus we set $\lambda>1$ in this paper. The constant $\gamma= \pm 1$. When $\gamma=1$, the black hole only has a single horizon $r_{h}$, which is the root of the equation $f\left(r_{h}\right)=0$. The function $f(r)$ for this case is given in Figure 1, which is similar to the Schwarzschild-AdS black hole. For $\gamma=-1$, the black hole is very similar to the Reissner-Nordstrom-AdS black hole. The function $f(r)$ for this case is given in Figure 2. The black hole event horizon $r_{h}$ is the largest root of the equation $f\left(r_{h}\right)=0$.

At the event horizon, the Hawking temperature and BHE can be written as

$$
\begin{aligned}
& T=\frac{1}{4 \pi} f^{\prime}\left(r_{h}\right)=\frac{1}{4 \pi}\left(\frac{1}{r_{h}}-r_{h} \Lambda+\frac{\gamma(\lambda-1) Q^{2}}{r_{h}^{\lambda+1}}\right), \\
& S=\pi r_{h}^{2} .
\end{aligned}
$$

The chemical potential in this black hole is

$$
\Phi=-\gamma \frac{Q}{r_{h}^{\lambda-1}} .
$$




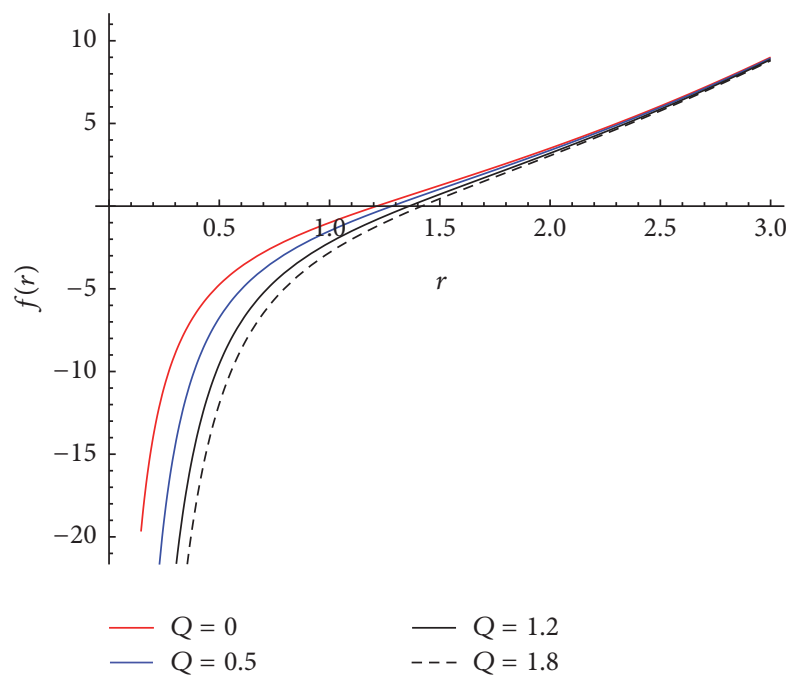

FIgURE 1: The figure shows $f(r)$ versus $r$ for $\gamma=1$ for varying $Q$. Here $\lambda=2.4, \Lambda=-3$, and $M=1.5$.

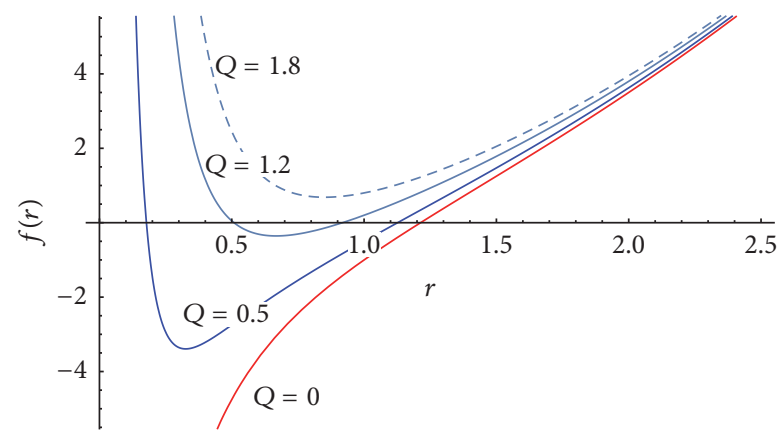

Figure 2: The figure shows $f(r)$ versus $r$ for $\gamma=-1$ for varying $Q$. Here $\lambda=2.4, \Lambda=-3$, and $M=1.5$.

We can check the first law of the black hole, which is given by

$$
d M=T d S+\Phi d Q
$$

There have been some works to study the thermodynamics and phase transitions of black holes in Lorentz breaking massive gravity [45-48].

2.2. Van der Waals-Like Phase Transition of BekensteinHawking Entropy. In this subsection, we focus on the VDW phase transition of BHE. Substituting (8) into (7) and eliminating the parameter $r_{h}$, one can get the relation between the Hawking temperature T and BHE $S$ of the AdS black holes in massive gravity as

$T$

$$
=\frac{S^{(1 / 2)(-\lambda-1)}\left(\gamma \pi^{\lambda / 2+1}(\lambda-1) Q-\Lambda S^{\lambda / 2+1}+\pi S^{\lambda / 2}\right)}{4 \pi^{3 / 2}} .
$$

This is the state equation of the AdS black hole thermodynamics system in massive gravity. Using (11), we investigate the phase diagram of the AdS black holes in massive gravity.

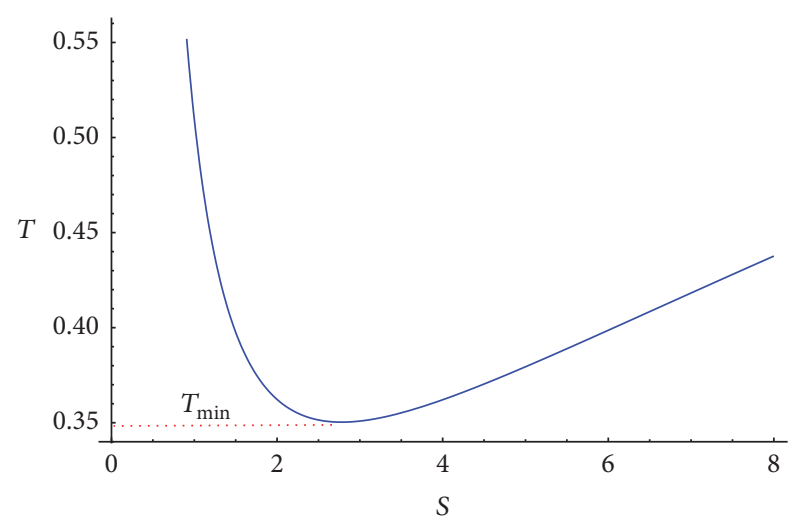

FIgure 3: The figure shows $T$ versus $S$ for $\gamma=1$. Here $\lambda=2.4$, $\Lambda=-3$, and $Q=0.3$.

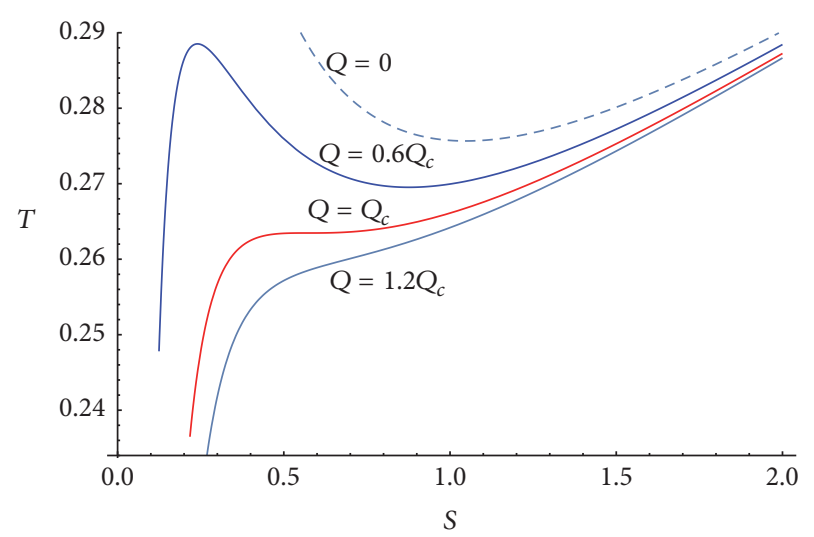

FIgUre 4: The figure shows $T$ versus $S$ for $\gamma=-1$ for varying $Q$. Here $\lambda=2.4, \Lambda=-3$. The top dashed curve is at $Q=0$ and the rest have $Q=0.6 Q_{c}, Q_{c}, 1.2 Q_{c}$, where $Q_{c}=0.12346$.

The temperature $T$ is plotted as a function of the BHE $S$ in Figures 3 and 4 for $\gamma=1$ and $\gamma=-1$, respectively. In Figure 3, the temperature is plotted for $\gamma=1$ where only one event horizon exists. This behavior of temperature is very similar to the behavior in the Schwarzschild-AdS black hole. That is to say, there is a minimum temperature $T_{\min }$ which divides the thermodynamics systems into small and large black holes. It is shown that, above the minimum temperature, small and large black holes coexist. In fact, this behavior will break for there is a first-order transition, which is similar to the Hawking-Page thermodynamic transition in [49].

In Figure 4, the temperature is plotted for $\gamma=-1$ where event horizon and inner horizon exist. Various values of $Q$ are used to plot the relations between the temperature and horizons. The top curve corresponds to $Q=0$. The system of this case is similar to the case $\gamma=1$ described above. When the scalar charge $Q$ increases, the temperature has two turning points. Further increasing of the scalar charge $Q$ makes these two turning points merge to one. It is shown that there exists a critical point $Q_{c}$. Above the critical point, the curve does not have any turning points. Thus we find that the phase structure is very similar to that of the Van der Waals gas-fluid phase transition. It should be noted that we 

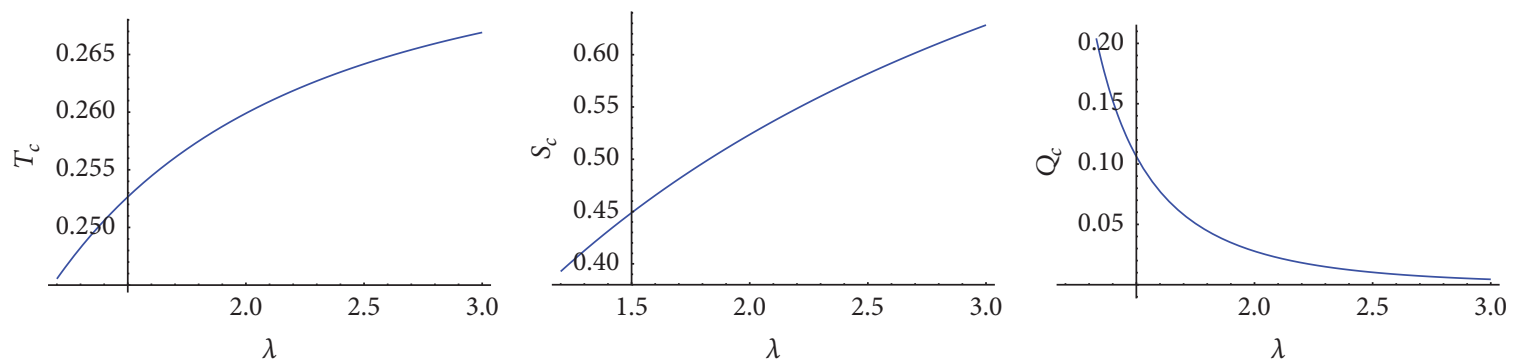

FIgURE 5: The figure shows the critical temperature $T_{c}$, the critical entropy $S_{c}$, and the critical charge $Q_{c}$ versus $\lambda$ for $\gamma=-1$. Here $\Lambda=-3$.

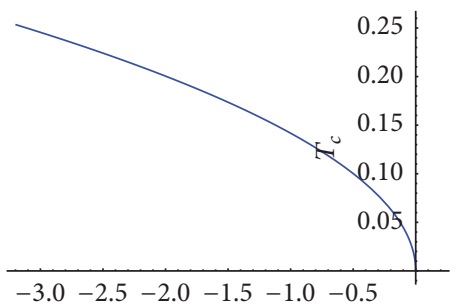

$\Lambda$

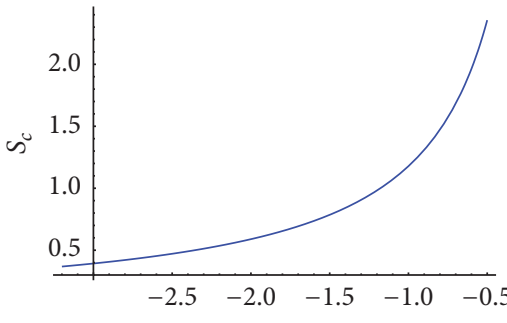

$\Lambda$

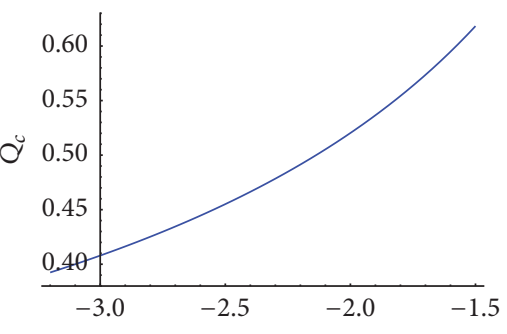

$\Lambda$

Figure 6: The figure shows the critical temperature $T_{c}$, the critical entropy $S_{c}$, and the critical charge $Q_{c}$ versus $\Lambda$ for $\gamma=-1$. Here $\lambda=1.2$.

mainly concentrate on this type of phase structure in this paper. Furthermore using the definition of the specific heat capacity $C_{\mathrm{Q}}$, that is,

$$
C_{Q}=T\left(\frac{\partial S}{\partial T}\right)_{Q}
$$

one can see that the specific heat capacity is divergent at the critical point and it is obvious that this phase transition is a SPT. At this critical point, the critical charge $Q_{c}$ and critical entropy $S_{c}$ can be obtained by the following equations:

$$
\left(\frac{\partial T}{\partial S}\right)_{Q_{c}, S_{c}}=\left(\frac{\partial^{2} T}{\partial S^{2}}\right)_{Q_{c}, S_{c}}=0
$$

After some calculation and using (11), $Q_{c}, S_{c}$, and the corresponding $T_{c}$ can be also got as

$$
\begin{aligned}
Q_{c} & =-\frac{2(-\lambda /(\lambda+2) \Lambda)^{\lambda / 2}}{\gamma(\lambda+2)\left(\lambda^{2}-1\right)}, \\
S_{c} & =-\frac{\pi \lambda}{(\lambda+2) \Lambda}, \\
T_{c} & =\frac{\lambda}{2 \pi(\lambda+1) \sqrt{-\lambda /(\lambda+2) \Lambda}} .
\end{aligned}
$$

Obviously, these critical parameters depend only on the internal parameters of the systems $\lambda$ and $\Lambda$. One can also see that $T_{c}$ and $S_{c}$ increase with $\lambda$ and $Q_{c}$ decreases with $\lambda$ as shown in Figure $5 ; S_{c}$ and $Q_{c}$ increase with $\Lambda$ and $T_{c}$ decreases with $\lambda$ as shown in Figure 6 . So the results show that the parameter $\lambda$ promotes the thermodynamic system to reach the stable state.

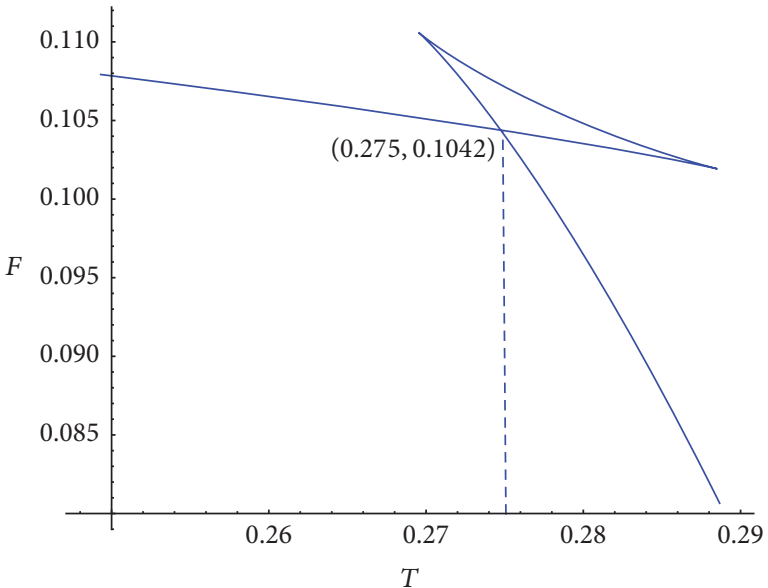

Figure 7: The figure shows $F$ versus $T$ for $\gamma=-1$. Here $\lambda=2.4$, $\Lambda=-3$, and $Q=0.6 Q_{c}$.

For the FPT, we will also check whether Maxwell's equal area law holds in this thermodynamic system. As is known to all, the first-order transition temperature $T^{*}$ plays a crucial role for Maxwell's equal area law. Thus in order to get $T^{*}$, we first plot the curve about the free energy with respect to the temperature $T$, where the free energy is defined by $F=M-T S$. The relation between $F$ with $T$ is plotted in Figure 7. One can see that there is a swallowtail structure, which corresponds to the unstable phase in Figure 8. The nonsmoothness of the junction implies that the phase transition is a FPT. The critical temperature $T^{*}$ is apparently given by the horizontal coordinate of the junction. From Figure 7, we get $T^{*}=0.2750$. Substituting this temperature $T^{*}$ into (11), one can obtain three values of the entropy, $S_{1}=0.1563$, 


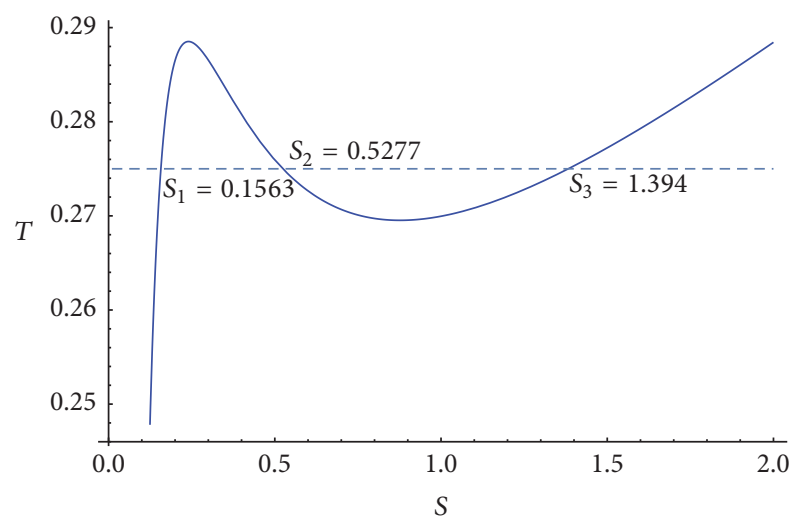

Figure 8: The figure shows $T$ versus $S$ for $\gamma=-1$. Here $\lambda=2.4$, $\Lambda=-3$, and $Q=0.6 Q_{c}$.

$S_{2}=0.5277$, and $S_{3}=1.394$. With these values, we can now check Maxwell's equal area law

$$
T^{*}\left(S_{3}-S_{1}\right)=\int_{S_{1}}^{S_{3}} T(S, Q) d S .
$$

After some calculation, we find that both the left and right sides of (17) are equal to 0.3404 exactly. Thus, our results show that Maxwell's equal area law is satisfied in this background.

For the SPT, we will study the critical exponent associated with the heat capacity definition in (12). Near the critical point, expanding the temperature as the very small amount $S-S_{c}$, we find

$$
\begin{aligned}
T= & T_{c}+\left(\frac{\partial T}{\partial S}\right)_{Q_{c}, S_{c}}\left(S-S_{c}\right)+\left(\frac{\partial^{2} T}{\partial S^{2}}\right)_{Q_{c}, S_{c}}\left(S-S_{c}\right)^{2} \\
& +\left(\frac{\partial^{3} T}{\partial S^{3}}\right)_{Q_{c}, S_{c}}\left(S-S_{c}\right)^{3}+o\left(S-S_{c}\right)^{4}
\end{aligned}
$$

Using (13), the second and third terms vanish. Then using (11), (14), and (15), we get

$$
T-T_{c}=\frac{\lambda}{16 \pi^{4}(-\lambda /(\lambda+2) \Lambda)^{7 / 2}}\left(S-S_{c}\right)^{3} .
$$

With the definition of the heat capacity (12), we further get $C_{\mathrm{Q}} \sim\left(T-T_{c}\right)^{-2 / 3}$. So one can find that the critical exponent of the heat capacity is $-2 / 3$, which is the same as the one from the mean field theory in Van der Waals gas-fluid system.

\section{Van der Waals-Like Phase Transition and Critical Phenomena of Holographic Entanglement Entropy}

In this section, our target is to explore whether the HEE has the similar VDW phase structure and critical phenomena as those of the BHE in massive gravity. For simplicity, here we only consider the case $\gamma=-1$. Now we will investigate whether there is VDW phase transition in the HEE-temperature phase plane.
Firstly, we review some basic knowledge about HEE. For detailed introduction of HEE, one can refer to $[18,19]$. For a given quantum field theory described by a density matrix $\rho$, HEE for a region $A$ and its complement $B$ are

$$
S_{A}=-\operatorname{Tr}_{A}\left(\rho_{A} \ln \rho_{A}\right)
$$

where $\rho_{A}$ is the reduced density matrix. However, it is usually not easy to get this quantity in quantum field theory. Fortunately according to AdS/CFT correspondence, $[18,19]$ propose a very simple geometric formula for calculating $S_{A}$ for static states with the area of a bulk minimal surface as $\partial A$; that is,

$$
S_{A}=\frac{\operatorname{Area}\left(\gamma_{A}\right)}{4}
$$

where $\gamma_{A}$ is the codimension-2 minimal surface according to boundary condition $\partial \gamma_{A}=\partial A$.

Subsequently using definition (21), we will calculate the HEE and study the corresponding phase transition. It is noted that the space on the boundary is spherical in the AdS black hole in massive gravity and the volume of the space is finite. Thus in order to avoid the HEE to be affected by the surface that wraps the event horizon, we will choose a small region as $A$. More precisely, as done in [23, 25-27], we choose region $A$ to be a spherical cap on the boundary given by $\varphi \leq \varphi_{0}$. Here the area can be written as

$$
A=2 \pi \int_{0}^{\varphi_{0}} \Theta(r(\varphi), \varphi) d \varphi
$$

$$
\Theta=r \sin \varphi \sqrt{\frac{\left(r^{\prime}\right)^{2}}{f(r)}+r^{2}},
$$

where $r^{\prime}=d r / d \varphi$. Then according to the Euler-Lagrange equation, one can get the equation of motion of $r(\varphi)$; that is,

$$
\begin{aligned}
0= & r^{\prime}(\varphi)^{2}\left[\sin \varphi r(\varphi)^{2} f^{\prime}(r)-2 \cos \varphi r^{\prime}(\varphi)\right] \\
& -2 r(\varphi) f(r)\left[r(\varphi)\left(\sin \varphi r^{\prime \prime}(\varphi)+\cos \varphi r^{\prime}(\varphi)\right)\right. \\
& \left.-3 \sin \varphi r^{\prime}(\varphi)^{2}\right]+4 \sin \varphi r(\varphi)^{3} f(r)^{2} .
\end{aligned}
$$

After using the boundary conditions $r^{\prime}(0)=0, r(0)=r_{0}$, we can get the numeric result of $r(\varphi)$.

It is worth noting that the HEE should be regularized by subtracting off the HEE in pure AdS, because the values of the HEE in (21) are divergent at the boundary. Now let us label the regularized HEE as $\delta S$. Here we choose the size of the boundary region to be $\varphi_{0}=0.10,0.16$ and set the UV cutoff in the dual field theory to be $r(0.099)$ and $r(0.159)$, respectively. The numeric results are shown in Figures 9 and 10. One can see that, for a given scalar charge $Q$, the relation between the HEE and temperature is similar to that between the BHE and temperature. That is to say, the AdS black holes thermodynamic system with the HEE undergoes the FPT and SPT one after another as the scalar charge $Q$ increases step by step. 


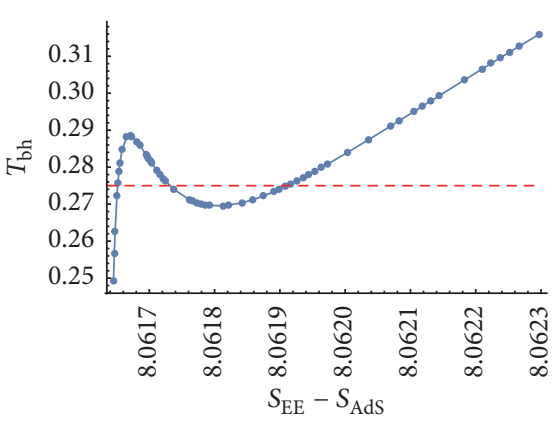

(a)

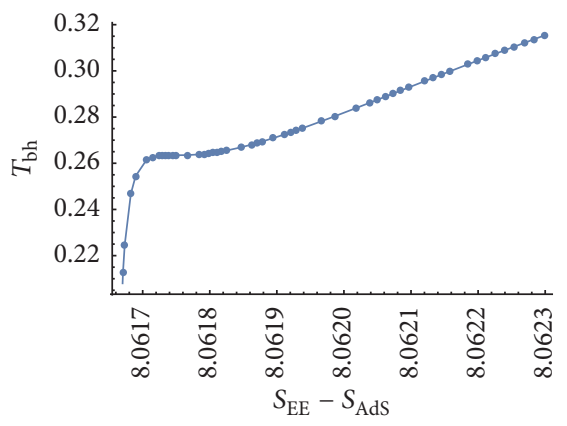

(b)

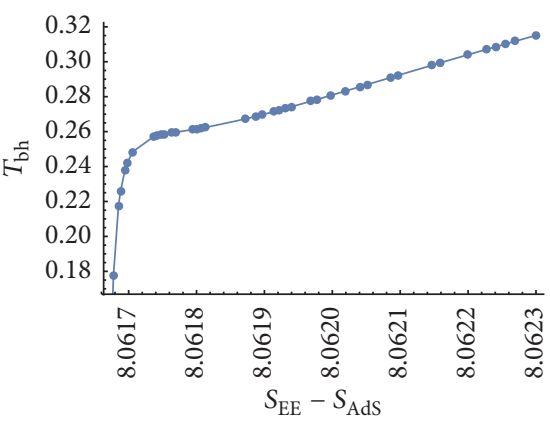

(c)

Figure 9: The figure shows $T$ versus $\delta S$ for $\gamma=-1, \varphi_{0}=0.10$. Here $\lambda=2.4, Q=0.6 Q_{c}, Q_{c}, 1.2 Q_{c}$ from (a) to (c).

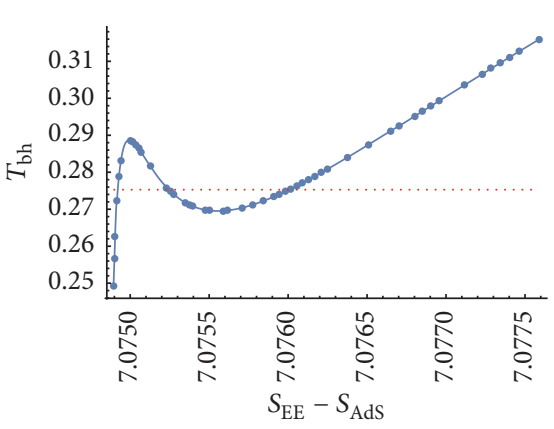

(a)

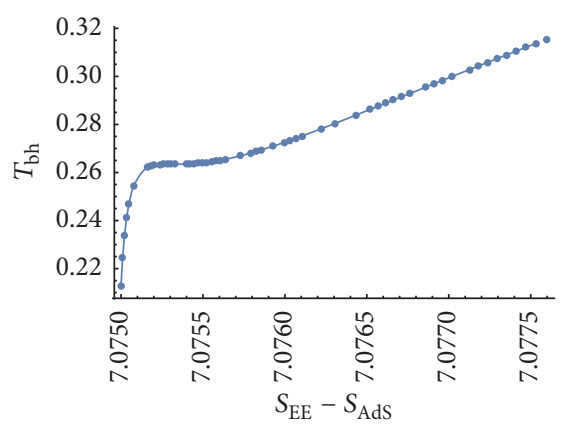

(b)

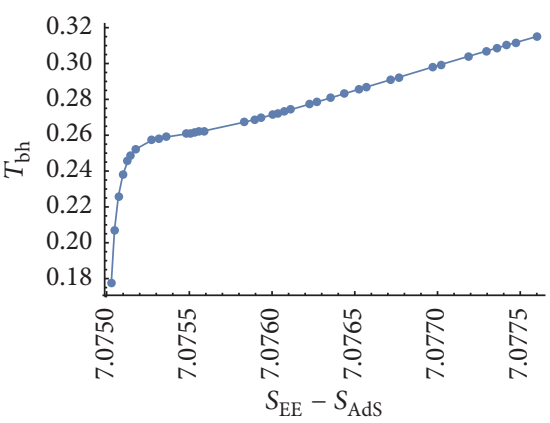

(c)

FIGURE 10: The figure shows $T$ versus $\delta S$ for $\gamma=-1, \varphi_{0}=0.16$. Here $\lambda=2.4, Q=0.6 Q_{c}, Q_{c}, 1.2 Q_{c}$ from (a) to (c).

For the FPT of the HEE, we now check whether Maxwell's equal area law is satisfied. Firstly, we get the interpolating function of the temperature $T(\delta S)$ using the data obtained numerically. At the first-order phase transition point, we get the smallest and largest roots for the equation $T(\delta S)=T^{*}$, which are $\delta S_{1}=8.06165, \delta S_{3}=8.06191$ for $\varphi_{0}=0.10$ and $\delta S_{1}=7.07492, \delta S_{3}=7.07599$ for $\varphi_{0}=0.16$. Then using these values and the equal area law

$$
T^{*}\left(\delta S_{3}-\delta S_{1}\right)=\int_{\delta S_{1}}^{\delta S_{3}} T(\delta S) d S,
$$

we find the left side equals $0.00007137,0.00029425$ and the right side equals $0.00007135,0.00029446$ for $\varphi_{0}=0.10,0.16$, respectively. Obviously both the left and the right sides are equal within our numerical accuracy and the relative errors are less than $0.022 \%, 0.071$ for $\varphi_{0}=0.10,0.16$, respectively.

Now let us consider the critical exponent of the SPT in the HEE-temperature phase plane. Here comparing with the definition of specific heat capacity $C_{Q}$ in (12), one can also define a specific heat capacity for the $\mathrm{HEE}$ as

$$
C_{Q}^{\prime}=T\left(\frac{\partial \delta S}{\partial T}\right)_{Q} .
$$

Then providing a similar relation of the critical points that in (13) is also working and using (25), we can get the critical exponent of SPT of in the HEE-temperature phase. Here we employ the logarithm of the quantities $T-T_{c}, \delta S-\delta S_{c}$. The relation between $\log \left|T-T_{c}\right|$ and $\log \left|\delta S-\delta S_{c}\right|$ is plotted in Figure 11. The analytical results of these straight lines can also be fitted, which are for $\varphi_{0}=0.10$,

$$
\log \left|T-T_{c}\right|=23.469+3.00654 \log \left|\delta S-\delta S_{c}\right|,
$$

and for $\varphi_{0}=0.16$,

$$
\log \left|T-T_{c}\right|=19.2089+3.00477 \log \left|\delta S-\delta S_{c}\right| .
$$

The results show that the slopes are all around 3 and the relative errors are less than $0.218 \%, 0.159 \%$ for $\varphi_{0}=0.10$, 0.16 , respectively, which are consistent with that of the BHE. Then one can find that the critical exponent of the specific heat capacity $C_{Q}^{\prime}$ is also approximately $-2 / 3$. That is to say, the HEE has the same SPT behavior as that of the BHE. Both of them are consistent with the result in the mean field theory of VDW gas-fluid system.

\section{Conclusions}

In this paper, we have investigated the VDW phase transition with the use of HEE as a probe. Firstly, we investigated the phase diagrams of the BHE in the $T-S$ phase plane and found that the phase structure depends on the scalar charge $Q$ and the parameter $\gamma$ of the AdS black holes in this massive gravity. For the case that $\gamma=1$ or $Q=0$, we found that there always exists the Hawking-Page-like phase transition in this thermodynamic system, while for the case $\gamma=-1$, we found 


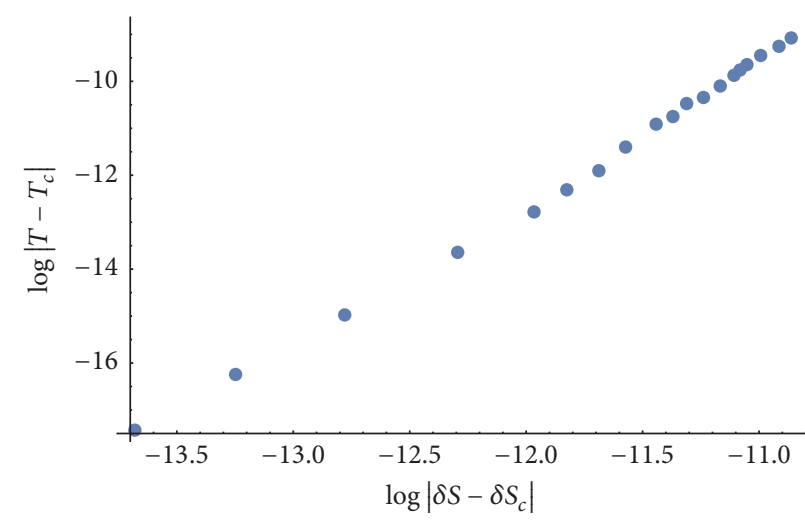

(a)

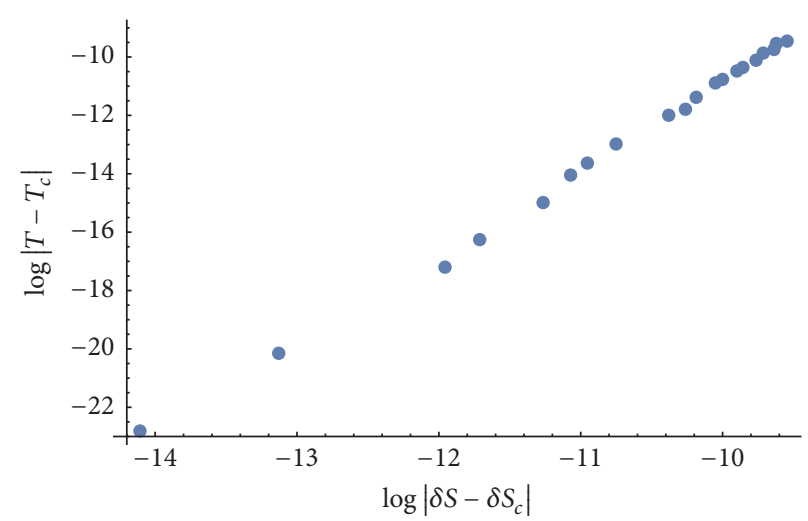

(b)

FIgURE 11: The figure shows $\log \left|T-T_{c}\right|$ versus $\log \left|\delta S-\delta S_{c}\right|$ for $\gamma=-1$. Here $\lambda=2.4, Q=Q_{c}$, where $\varphi_{0}=0.10$ in (a) and $\varphi_{0}=0.16$ in (b).

for the small scalar charge $Q$, there is always an unstable black hole thermodynamic system interpolating between the small stable black hole system and large stable black hole system. The thermodynamic transition for the small hole to the large hole is a first-order transition and Maxwell's equal area law is valid. As the scalar charge $Q$ increases to the critical value $Q_{c}$ in this space-time, the unstable black hole merges into an inflection point. We found there is a SPT at the critical point. When the scalar charge is larger than the critical charge, the black hole is stable always. That is to say, we found that there exists the VDW gas-fluid phase transition in the $T-S$ phase plane of the AdS black hole in massive gravity.

The more interesting thing is that we found the HEE also exhibits the VDW phase structure in the $T-\delta S$ plane when $\gamma=-1$ and the scalar charge $Q \neq 0$. In order to confirm this result, we further showed that Maxwell's equal area law is satisfied and the critical exponent of the specific heat capacity is consistent with that of the mean field theory of the VDW gas-fluid system for the HEE system. These results show that the phase structure of HEE is similar to that of BHE and the $\mathrm{HEE}$ is really a good probe to the phase transition of AdS black holes in Lorentz breaking massive gravity. This also implies that HEE and BHE exhibit some potential underlying relationship.

\section{Conflicts of Interest}

The authors declare that they have no conflicts of interest.

\section{Acknowledgments}

The authors are grateful to De-Fu Hou and Hong-Bao Zhang for their instructive discussions. This work is supported by the National Natural Science Foundation of China (Grant no. 11365008, Grant no. 11465007, and Grant no. 61364030).

\section{References}

[1] C. H. Bennett, G. Brassard, C. Crépeau, R. Jozsa, A. Peres, and W. K. Wootters, "Teleporting an unknown quantum state via dual classical and Einstein-Podolsky-Rosen channels," Physical Review Letters, vol. 70, no. 13, pp. 1895-1899, 1993.
[2] C. H. Bennett and D. P. Divincenzo, "Quantum information and computation," Nature, vol. 404, no. 6775, pp. 247-255, 2000.

[3] M. A. Nielsen and I. L. Chuang, Quantum Computation and Quantum Information, Cambridge University Press, Cambridge, UK, 1st edition, 2000.

[4] J. Preskill, "Quantum information and physics: some future directions," Journal of Modern Optics, vol. 47, no. 2-3, pp. 127$137,2000$.

[5] T. J. Osborne and M. A. Nielsen, "Entanglement, quantum phase transitions, and density matrix renormalization," Quantum Information Processing, vol. 1, no. 1-2, pp. 45-53, 2002.

[6] P. Zanardi and X. Wang, "Fermionic entanglement in itinerant systems," Journal of Physics. A. Mathematical and General, vol. 35, no. 37, pp. 7947-7959, 2002.

[7] X.-X. Zeng and W.-B. Liu, "Holographic thermalization in Gauss-Bonnet gravity," Physics Letters. B. Particle Physics, Nuclear Physics and Cosmology, vol. 726, no. 1-3, pp. 481-487, 2013.

[8] X.-X. Zeng, X.-M. Liu, and W.-B. Liu, "Holographic thermalization with a chemical potential in Gauss-Bonnet gravity," Journal of High Energy Physics, vol. 2014, no. 3, article 031, 2014.

[9] X. X. Zeng, D. Y. Chen, and L. F. Li, "Holographic thermalization and gravitational collapse in a spacetime dominated by quintessence dark energy," Physical Review D, vol. 91, no. 4, Article ID 046005, 2015.

[10] X.-X. Zeng, X.-M. Liu, and W.-B. Liu, "Holographic thermalization in noncommutative geometry," Physics Letters. B. Particle Physics, Nuclear Physics and Cosmology, vol. 744, pp. 48-54, 2015.

[11] X. X. Zeng, X. Y. Hu, and L. F. Li, "Effect of phantom dark energy on holographic thermalization," Chinese Physics Letters, vol. 34, no. 1, Article ID 010401, 2017.

[12] R. Cai, X. Zeng, and H. Zhang, "Influence of inhomogeneities on holographic mutual information and butterfly effect," Journal of High Energy Physics, vol. 2017, no. 7, 2017.

[13] R. Cai, L. Li, L. Li, and R. Yang, "Introduction to holographic superconductor models," Science China Physics, Mechanics \& Astronomy, vol. 58, no. 6, pp. 1-46, 2015.

[14] L. Amico, R. Fazio, A. Osterloh, and V. Vedral, "Entanglement in many-body systems," Reviews of Modern Physics, vol. 80, no. 2, pp. 517-576, 2008. 
[15] N. Laflorencie, "Quantum entanglement in condensed matter systems," Physics Reports. A Review Section of Physics Letters, vol. 646, pp. 1-59, 2016.

[16] L. Susskind and J. Uglum, "Black hole entropy in canonical quantum gravity and superstring theory," Physical Review. D. Third Series, vol. 50, no. 4, pp. 2700-2711, 1994.

[17] S. N. Solodukhin, "Entanglement entropy of black holes," Living Reviews in Relativity, vol. 14, article 8, 2011.

[18] S. Ryu and T. Takayanagi, "Holographic derivation of entanglement entropy from the anti-de Sitter space/conformal field theory correspondence," Physical Review Letters, vol. 96, no. 18, Article ID 181602, 181602, 4 pages, 2006.

[19] S. Ryu and T. Takayanagi, "Aspects of holographic entanglement entropy," Journal of High Energy Physics. A SISSA Journal, no. 8, 045, 48 pages, 2006.

[20] C. V. Johnson, "Large $N$ phase transitions, finite volume, and entanglement entropy," Journal of High Energy Physics, vol. 2014, article 47, 2014.

[21] E. Caceres, P. H. Nguyen, and J. F. Pedraza, "Holographic entanglement entropy and the extended phase structure of STU black holes," Journal of High Energy Physics, vol. 2015, no. 9, article 184, 2015.

[22] P. H. Nguyen, "An equal area law for holographic entanglement entropy of the AdS-RN black hole," Journal of High Energy Physics, vol. 15, no. 12, article 139, 2015.

[23] X. X. Zeng, H. Zhang, and L. F. Li, "Phase transition of holographic entanglement entropy in massive gravity," Physics Letters B, vol. 756, pp. 170-179, 2016.

[24] A. Dey, S. Mahapatra, and T. Sarkar, "Thermodynamics and entanglement entropy with Weyl corrections," Physical Review D - Particles, Fields, Gravitation and Cosmology, vol. 94, no. 2, Article ID 026006, 2016.

[25] X.-X. Zeng, X.-M. Liu, and L.-F. Li, "Phase structure of the Born-Infeld-anti-de Sitter black holes probed by non-local observables," European Physical Journal C, vol. 76, no. 11, article 616, 2016.

[26] X.-X. Zeng and L.-F. Li, "Holographic Phase Transition Probed by Nonlocal Observables," Advances in High Energy Physics, vol. 2016, Article ID 6153435, 2016.

[27] X. X. Zeng and L. F. Li, "Van der waals phase transition in the framework of holography," Physics Letters B, vol. 764, pp. 100108, 2017.

[28] J.-X. Mo, G.-Q. Li, Z.-T. Lin, and X.-X. Zeng, "Revisiting van der Waals like behavior of $f(R)$ AdS black holes via the two point correlation function," Nuclear Physics B, vol. 918, pp. 11-22, 2017.

[29] S. He, L. F. Li, and X. X. Zeng, "Holographic van der waals-like phase transition in the Gauss-Bonnet gravity," Nuclear Physics $B$, vol. 915, pp. 243-261, 2017.

[30] X. Zeng and Y. Han, "Holographic van der waals phase transition for a hairy black hole," Advances in High Energy Physics, vol. 2017, Article ID 2356174, 8 pages, 2017.

[31] H.-L. Li, S.-Z. Yang, and X.-T. Zu, "Holographic research on phase transitions for a five dimensional AdS black hole with conformally coupled scalar hair," Physics Letters, Section B: Nuclear, Elementary Particle and High-Energy Physics, vol. 764, pp. 310-317, 2017.

[32] M. Cadoni, E. Franzin, and M. Tuveri, "Van der Waals-like behaviour of charged black holes and hysteresis in the dual QFTs," Physics Letters. B. Particle Physics, Nuclear Physics and Cosmology, vol. 768, pp. 393-396, 2017.
[33] B. P. Abbott et al., "Observation of gravitational waves from a binary black hole merger," Physical Review Letters, vol. 116, Article ID 061102, 2016.

[34] M. Fierz and W. Pauli, "On relativistic wave equations for particles of arbitrary spin in an electromagnetic field," Proceedings of the Royal Society of London Series A: Mathematical and Physical Sciences, vol. 173, no. 953, pp. 211-232, 1939.

[35] D. G. Boulware and S. Deser, "Can gravitation have a finite range?” Physical Review D, vol. 6, no. 12, pp. 3368-3382, 1972.

[36] C. de Rham and G. Gabadadze, "Generalization of the FierzPauli action," Physical Review D, vol. 82, Article ID 044020, 2010.

[37] C. De Rham, G. Gabadadze, and A. J. Tolley, "Resummation of massive gravity," Physical Review Letters, vol. 106, no. 23, Article ID 231101, 2011.

[38] D. Vegh, "Holography without translational symmetry," 2013, https://arxiv.org/abs/1301.0537.

[39] C. de Rham, "Massive gravity," Living Reviews in Relativity, vol. 17, no. 7, 2014.

[40] K. Hinterbichler, "Theoretical aspects of massive gravity," Reviews of Modern Physics, vol. 84, no. 2, 2012.

[41] S. L. Dubovsky, "Phases of massive gravity," Journal of High Energy Physics, article 076, 2004.

[42] V. A. Rubakov and P. G. Tinyakov, "Infrared-modified gravities and massive gravitons," Physics-Uspekhi, vol. 51, no. 8, pp. 759792, 2008.

[43] M. V. Bebronne and P. G. Tinyakov, "Black hole solutions in massive gravity," Journal of High Energy Physics, vol. 09, no. 04, article 100, 2008.

[44] D. Comelli, F. Nesti, and L. Pilo, "Stars and (furry) black holes in Lorentz breaking massive gravity," Physical Review D - Particles, Fields, Gravitation and Cosmology, vol. 83, no. 8, Article ID 084042, 2011.

[45] S. Fernando, "Phase transitions of black holes in massive gravity," Modern Physics Letters A. Particles and Fields, Gravitation, Cosmology, Nuclear Physics, vol. 31, no. 16, Article ID 1650096, 1650096, 19 pages, 2016.

[46] F. Capela and G. Nardini, "Hairy black holes in massive gravity: thermodynamics and phase structure," Physical Review D, vol. 86, no. 2, Article ID 024030, 12 pages, 2012.

[47] B. Mirza and Z. Sherkatghanad, "Phase transitions of hairy black holes in massive gravity and thermodynamic behavior of charged AdS black holes in an extended phase space," Physical Review D, vol. 90, no. 8, Article ID 084006, 2014.

[48] S. Fernando, "P-V criticality in AdS black holes of massive gravity," Physical Review D, vol. 94, no. 12, Article ID 124049, 2016.

[49] S. W. Hawking and D. N. Page, "Thermodynamics of black holes in anti-de Sitter space," Communications in Mathematical Physics, vol. 87, no. 4, pp. 577-588, 1982/83. 

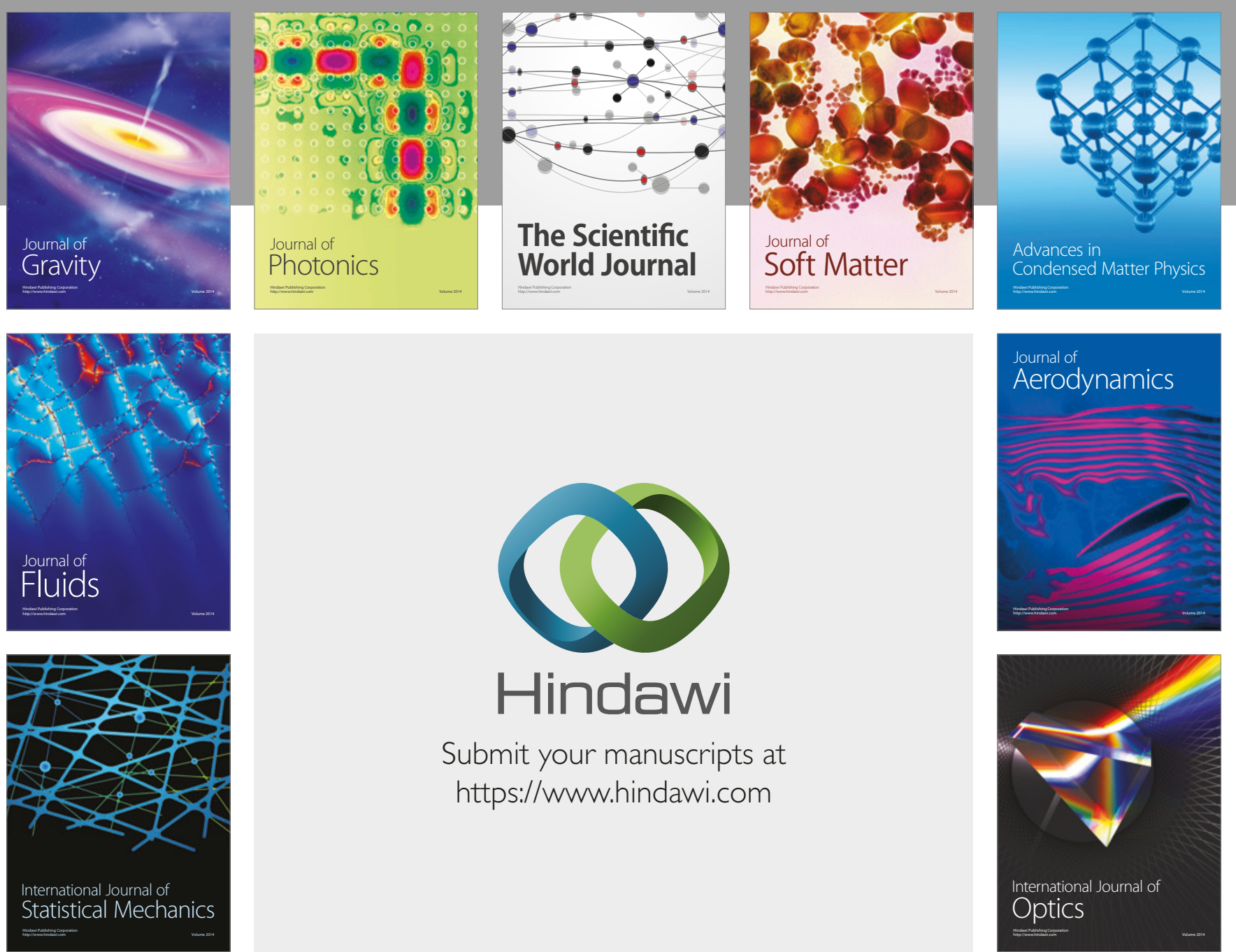

Submit your manuscripts at

https://www.hindawi.com
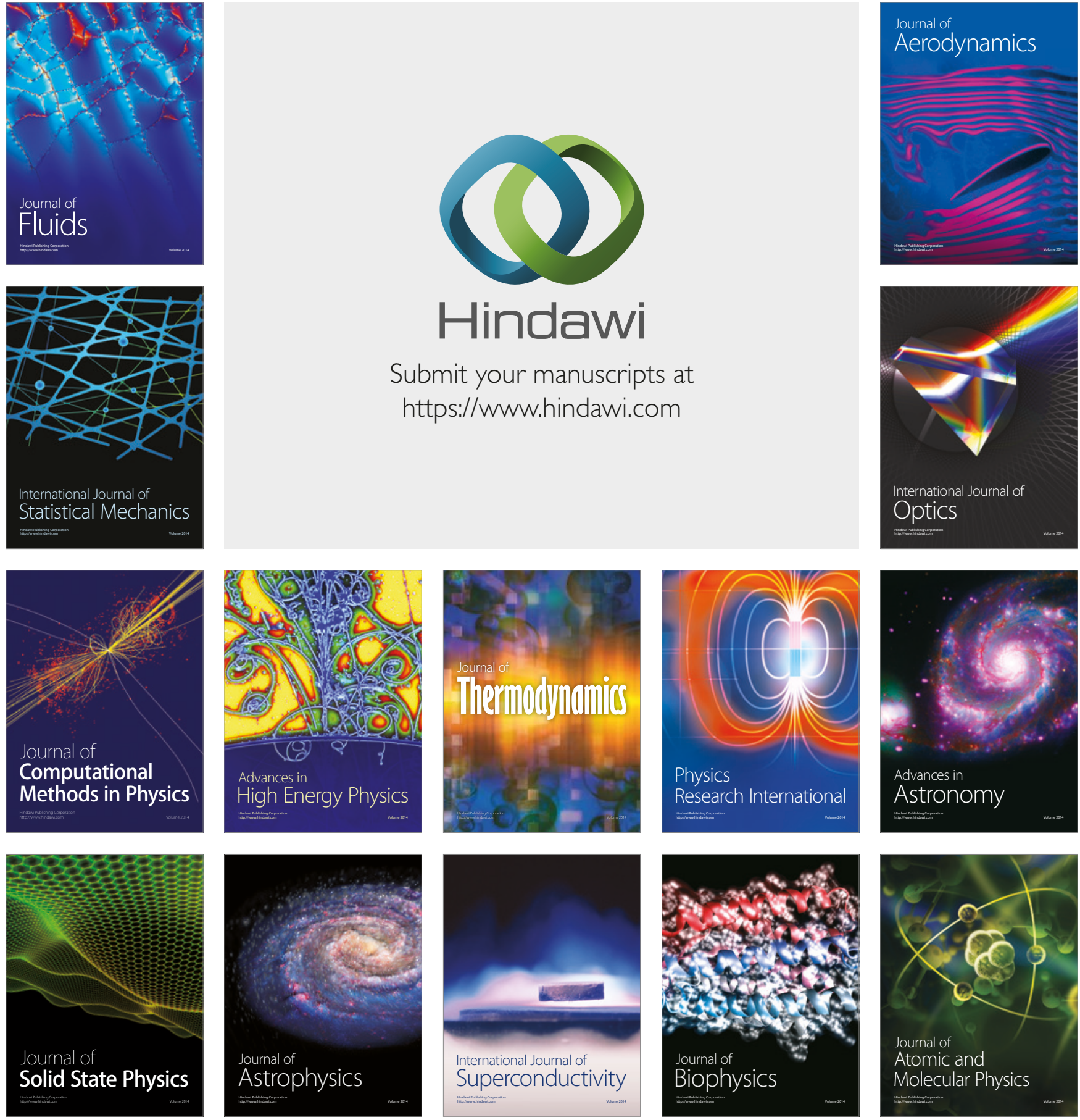